\title{
Scanned aperture polarized light microscope with liquid crystal compensator
}

\author{
M. Shribak, and R. Oldenbourg \\ Marine Biological Laboratory, Woods Hole, MA, 02543
}

We have been developing a polarized light microscope and sensitive technique for recording images of birefringent specimens with high spatial resolution and short measurement intervals. Images show the magnitude and orientation distributions of the in-plane birefringence of the focused region in the specimen [1]. The technique can be implemented on most research grade polarizing microscopes by adding a pair of liquid crystal retarders forming a universal compensator, a circular analyzer, an electronic camera, analog and digital control electronics and custom software [2]. We have now introduced an aperture scanning device for combining the traditional methods of orthoscopic and conoscopic observations of birefringent objects. Using the scanning device, both types of measurements can be performed simultaneously on all objects within the field of view. The scanning device, which is placed in the front aperture plane of the condenser lens, consists of two parts, a universal compensator and a spatial light modulator. An example of the microscope polarization scheme including the aperture scanning device is shown in Fig. 1.

For measuring the in-plane birefringence in the focused specimen region we use an open aperture mask and take one image of the specimen with settings $\alpha=\lambda / 4$ and $\beta=\lambda / 2$ of the variable retarder plates LC1 and LC2. Additional images are recorded with a small swing value added or subtracted in turn to the retardance of each plate. The recorded images are processed to get pictures of in-plane retardance magnitude and azimuth of the slow axis. For faster, but less sensitive measurements it is possible to reduce the set of raw images; and for removing the influence of background retardance, drift of liquid crystal retardance and intensity fluctuations of the light source we use a specially designed correction procedure [3]. A further modification of the universal compensator, not discussed here, can be used to rectify polarization aberrations introduced by the microscope optics [4].

For exploring out-off-plane birefringence the Pol-Scope set up contains the additional spatial light modulator, implemented here as a liquid crystal aperture mask. Partial occlusion of the condenser aperture changes the direction of the central ray of the cone of light converging on the specimen. So we can obtain the magnitude and azimuth images with different sets of illumination rays. In the current procedure illumination set with fully opened mask and four sets with quarter-opened masks are used. Calculated images of the out-of-plane direction of birefringence axis and azimuth of its projection on the focal plane are shown in Fig. 2. Here we present images of a biological object called an aster. An asters consists of nearly parallel arrays of microtubules, a stiff biopolymer, radiating from a common organizing center called a centrosome.

References

[1] R. Oldenbourg and G. Mei, New polarized light microscope with precision universal compensator. J. Microsc. 180: 140-147 (1995)..

[2] The commercial LC-PolScope is manufactured by Cambridge Research and Instrumentation, Woburn, MA, USA.

[3] M. Shribak, and R. Oldenbourg, Applied Optics, vol. 42, (2003), in print

[4] M. Shribak, S. Inoué and R. Oldenbourg, Optical Engineering, vol. 41, No. 5, p.943-954, (2002).

[5] The research was supported by the National Institutes of Health grant GM49210. Authors are thankful to Dr. S. Inoue for helpful discussion and comments of the work. 


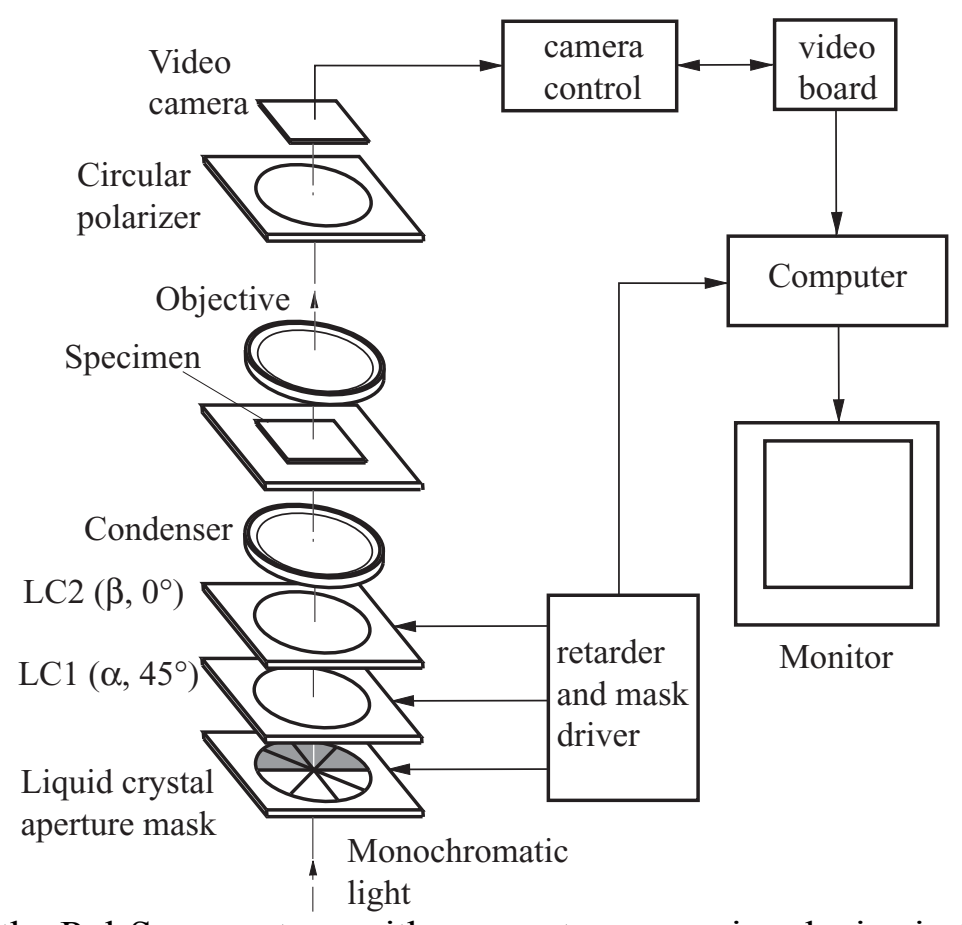

Fig.1. Schematics of the Pol-Scope set-up with an aperture scanning device in the illumination path. The liquid crystal aperture mask consists of a radially segmented variable retarder plate between two parallel linear polarizers. LC1 and LC2 are variable retarder plates forming together a universal compensator. $\alpha$ and $\beta$ denote the retardance of plates $\mathbf{L C 1}$ and $\mathbf{L C} 2$ with azimuths of their slow axis $45^{\circ}$ and $0^{\circ}$ respectively.

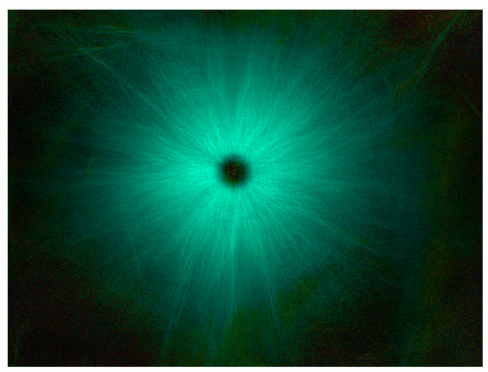

$\gamma, \mathrm{z}=0 \mu \mathrm{m}$

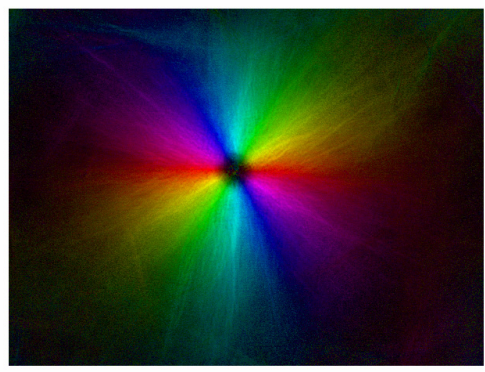

$\varphi, \mathrm{z}=0 \mu \mathrm{m}$
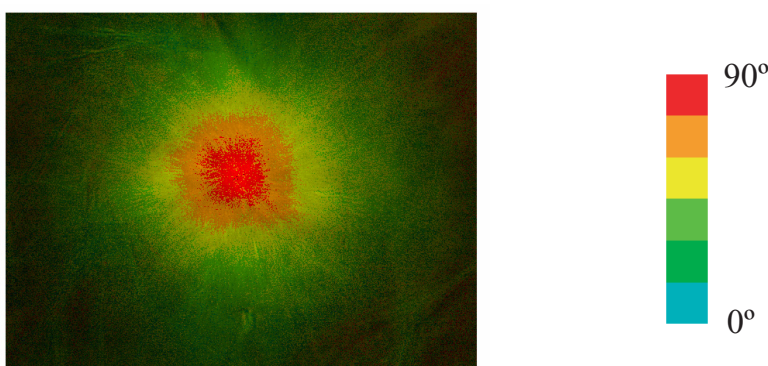

$\gamma, \mathrm{z}=7 \mu \mathrm{m}$
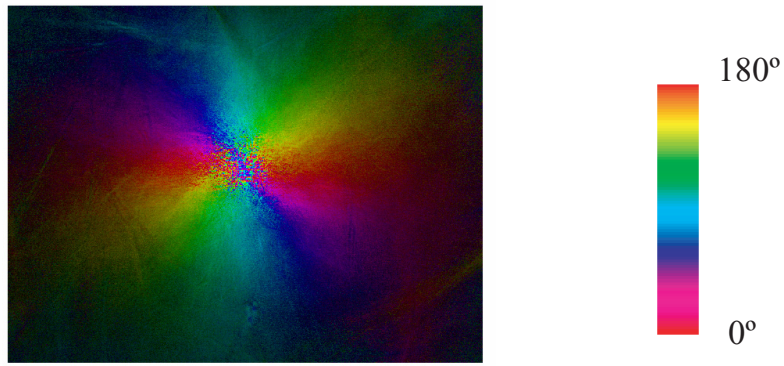

$\varphi, \mathrm{z}=7 \mu \mathrm{m}$

Fig.2. Calculated images of the out-of-plane direction of birefringence axis $\gamma$ and azimuth of its projection on the focal plane $\varphi$ for two axial positions $\mathbf{z}=0 \mu \mathrm{m}$ and $\mathbf{z}=7 \mu \mathrm{m}$ 